\title{
GPC-based Teleoperation for Delay Compensation and Disturbance Rejection in Image-guided Beating-heart Surgery
}

\author{
Meaghan Bowthorpe, IEEE Student Member ${ }^{1}$, Abril Álvarez García ${ }^{2}$, and Mahdi Tavakoli, IEEE Member ${ }^{1}$
}

\begin{abstract}
Beating-heart surgery is not currently possible for most surgical procedures as it requires superhuman skill to manually track the heart's motion while performing a surgical task. However, if a surgical tool could track the motion of the point of interest (POI) on the heart, then, with respect to the surgical tool tip the POI would appear stationary. Such a system can be created with a teleoperated surgical robot that is controlled to track the combination of the heart's and the surgeon's motion, as input through a separate user console. To develop such a system, the motion of the heart is found in ultrasound images where the image acquisition introduces delays of approximately $40 \mathrm{~ms}$ and image processing further increases this delay. Directly using this delayed position measurement in the feedback control loop can lead to instability and poor tracking. The generalized predictive controller used in this work compensates for this time delay despite large disturbances with velocities up to $210 \mathrm{~mm} / \mathrm{s}$ and accelerations up to $3800 \mathrm{~mm} / \mathrm{s}^{2}$ caused by the moving heart.
\end{abstract}

\section{INTRODUCTION}

Cardiovascular disease is one of the leading causes of death worldwide [1]. Any new cardiac surgical techniques that increase the safety and reduce the recovery time will greatly benefit society. Currently, very few procedures are performed on a freely beating heart as the surgeon cannot manually track the motion of the heart, which is beating with velocities and accelerations up to $210 \mathrm{~mm} / \mathrm{s}$ and $3800 \mathrm{~mm} / \mathrm{s}^{2}$, respectively [2]. Instead, the heart is either mechanically stabilized or arrested. However, both methods have drawbacks. Mechanically stabilizing the heart is only effective on a localized area on the exterior surface of heart that is accessible through the chest cavity. The pressure or suction used to prevent motion can damage the heart tissue. Also, there will be some residual motion [3]. Arresting the heart after connecting the patient to a heart-lung machine can lead to long term cognitive loss [4] and increase the risk of stroke [5]. Complications may occur when the heart is restarted.

If the heart was made to appear stationary using a surgical robot to move the surgical tool in synchrony with the beating heart, these risks could be removed. Given a stabilized view of the heart, the surgeon can now perform a surgical task on

*This work was supported by the Natural Sciences and Engineering Research Council (NSERC) of Canada, the Canada Foundation for Innovation (CFI), and a Queen Elizabeth II Graduate Student Scholarship from the Government of Alberta.

${ }^{1}$ Meaghan Bowthorpe and Mahdi Tavakoli are with the Department of Electrical and Computer Engineering, University of Alberta, AB, Canada. meaghan.bowthorpe@ualberta.ca, mahdi.tavakoli@ualberta.ca

${ }^{2}$ Abril Álvarez García was a visiting student with the Department of Electrical and Computer Engineering, University of Alberta, AB, Canada from the Department of Electronics Engineering, Universidad de Guadalajara, Jalisco, Mexico. abril.alvarez.garcia@gmail.com

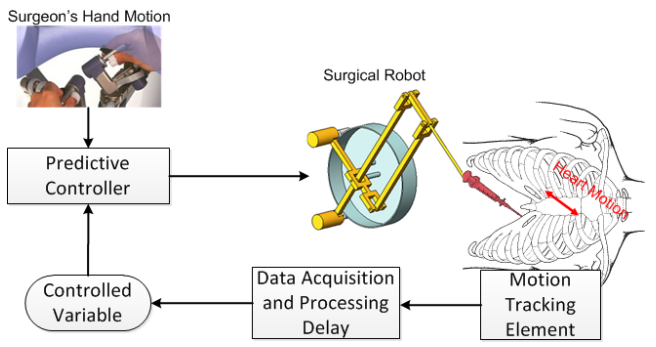

Fig. 1. An overview of a robot-assisted beating-heart surgical system.

a virtually stabilized though actually freely beating heart. In addition, the outcome of reconstructive procedures could be evaluated intra-operatively when improvements can be made. The goal of this research is to develop such a system that can be used to perform surgical procedures on both the exterior surface and the interior of the heart through teleoperation.

\section{PRIOR ART}

To make the surgical tool follow the heart's motion, two core elements are required. The first is a method of determining the location of the point of interest (POI) on the heart. ${ }^{1}$ The second is a position control law to make the surgical tool follow the motion of the POI found in the first element. As far as the first element, the location of the POI is found using some type of sensor data and can be divided into two categories: non-image-based methods and image-based methods. An important consideration is whether a time delay is introduced when acquiring and processing the raw sensor data to find the position of the POI (i.e., the first element). This time delay, if not compensated for by the control system (i.e., the second element), can lead to instability that may cause the surgical tool to collide with and puncture the heart. A diagram of a robot-assisted beating-heart surgical system is given in Fig. 1. Different motion tracking and control methods have been proposed in the literature and will be discussed in more detail in the following two subsections.

\section{A. HEART MOTION TRACKING METHODS}

The heart's motion is caused by respiratory-induced and heartbeat-induced motion. Some motion tracking methods can record both sources of motion, others only record the heartbeat-induced motion. Each method is tailored to a specific type of surgical procedure.

\footnotetext{
${ }^{1}$ For certain procedures, the POI's position is not tracked and thus this first element is not needed. This occurs when the surgical tool is forcecontrolled, for example in ablation, where the surgical tool must apply a constant force on the tissue [6], [7], [8], [9].
} 
1) NON-IMAGE-BASED METHODS: A non-imagebased method may be used for procedures performed on the exterior surface of the heart. For instance, the use of sonomicrometry crystals, small crystals that are sutured to the heart, is well suited to evaluating the surgical system [10], [11], [12], but is not practical in the operating room. In addition, significant computation is required to analyze the raw sensor data, making this method not suitable for real-time applications. Work is being done to reduce the computational burden with the goal of being able to use sonomicrometry crystals in real-time [11].

2) IMAGE-BASED METHODS: Image-based methods, be it from a camera or a medical imager, can capture the motion of the surgical tool and the POI as long as both are visible in the image. However, at times the surgical tool may occlude the POI [3]. Camera-based methods are used to track a POI on the exterior surface of the heart and can record both the respiratory-induced and heartbeat-induced motion, but they cannot be used for intracardiac procedures as they cannot visualize through the opaque blood pool. High-speed cameras $(500 \mathrm{~Hz}$ in [13] and $955 \mathrm{~Hz}$ in [14]) are proposed for coronary artery bypass graft $(\mathrm{CABG})$ procedures.

A main advantage of medical image-based methods is that they can track a POI on the exterior surface or interior of the heart. MRI images can track both respiratory- and heartbeat-induced motion, but cannot be acquired in realtime. However, ultrasound images only track the heartbeatinduced motion as the ultrasound probe must remain in contact with the patient's skin and will therefore move in synchrony with the respiratory-induced motion. On the bright side, ultrasound images can be acquired in real-time, tens of times per second, can visualize through blood, and are used in [15], [16], [17] for annuloplasty and pericardial effusion.

The focus of this paper is to develop a system that can aid in a surgical procedure performed on either the exterior surface or the interior of the heart. For intracardiac procedures, it is imperative to track the position of heart tissue based on ultrasound images.

\section{B. CONTROL METHODS}

Depending on the motion tracking method chosen, different control systems have been proposed to make the surgical tool follow the heart's motion. Some may need to compensate for the data acquisition and processing time delay, others may need to take the surgical robot's dynamics into account for a teleoperated system, and some may need to do both. The control methods that compensate for time delays can be separated into two main categories: predictive feedforward controllers and predictive feedback controllers.

1) PREDICTIVE FEEDFORWARD CONTROL: In the presence of data acquisition and processing delays, the heart will have moved by the time the position measurement of the POI is obtained. This creates the unusual requirement of estimating the heart's current position from past and current sensor data. Predictive feedforward control uses an estimate of the heart's current position as the desired position. To predict the current position of the POI, Bebek et al. timeshift the motion from the previous heart beat [10] and Yuen et al. use an extended Kalman filter based on a time-varying Fourier series [17]. Evidently, these methods are useful for hand-held instruments where there are no dynamics between the surgeon and the tool (in comparison to master-slave teleoperation, it is as if the master and the slave are combined into one tool). They are not suitable for teleoperation where the dynamics of the surgical robot (slave) must be accounted for in order to accurately follow the surgeon's motion.

2) PREDICTIVE FEEDBACK CONTROL: Predictive feedback controllers also estimate the heart's current position and additionally account for the slave robot's dynamics, which is necessary in a teleoperation setting. Ginhoux et al. used Generalized predictive control (GPC) to follow the periodic respiratory-induced motion and an adaptive disturbance predictor to follow the heartbeat-induced motion [13]. Bowthorpe et al. used a Smith predictor to stabilize the surgical system in the presence of long image processing and acquisition delays of approximately $150 \mathrm{~ms}$ to track the heartbeat-induced motion [15].

This paper focuses on the last method i.e. systems where both the image acquisition and processing delays and the surgical robot's dynamics must be accounted for. It expands on the work previously done by Ginhoux et al. [13] and Bowthorpe et al. [15], by aiming to develop a teleoperated system guided by ultrasound images that follows the heartbeat-induced motion of the POI for procedures performed on the exterior surface or interior of the heart. Hence, the image acquisition and processing delay is significantly larger than that in [13] where a $500 \mathrm{~Hz}$ camera as opposed to a $28 \mathrm{~Hz}$ ultrasound scanner is used. Importantly, by using a generalized predictive controller instead of a Smith predictor as in [15], disturbances originating from the heart's backand-forth motion can be rejected directly by the controller.

The rest of this paper is organized as follows. Section III reviews the generalized predictive control law and describes the algorithms used to develop a beating-heart surgical system. Section IV describes the experimental setup and results that validate the proposed algorithm. The concluding remarks are given in Section V.

\section{PREDICTIVE FEEDBACK CONTROL LAW}

This paper focuses on a surgical system that could be used for procedures performed on both the exterior surface and the interior of the heart. Hence, ultrasound images, which can visualize through blood, are used to track the beating-induced motion of the POI. This introduces an image acquisition and processing delay of approximately $40 \mathrm{~ms}$ and $100 \mathrm{~ms}$ [16], respectively. If this delay is not compensated for by the control law, there is a risk that the surgical tool will collide with and puncture the heart tissue. In addition, the surgical robot is teleoperated from a surgeon's console and hence the control system must consider the surgical robot's dynamics to ensure the surgeon's motions are accurately mimicked by the surgical robot. 
As mentioned previously, the position measurement of the surgical tool tip is delayed. While this measurement in principle can be obtained in real-time from an external position sensor, this measurement will be with respect the surgical tool's reference frame and not the image frame. Note that the goal is to control the surgical tool tip to follow the POI's motion, which is only known in the image frame. Although the transformation between the surgical tool's frame and the image frame can theoretically be calculated, in a clinical setting this is difficult to do. For instance, if the ultrasound probe moves, which is not rare in the operating room, the transformation will need to be recalculated. A more robust alternatively is to measure the surgical tool tip's position in the image frame directly, meaning that the position will not be available until after the image acquisition and processing delay, which the control system will need to compensate for.

\section{A. OVERVIEW OF GENERALIZED PREDICTIVE CON- TROL}

Generalized predictive control was chosen due to its ability to compensate for a time delay and to reject disturbances caused by the heart motion [18]. In GPC, the system is modeled by a controlled auto-regressive integrated moving average (CARIMA) equation - see (1) - that includes a time delay of $d$ samples [18]. An overview of GPC is shown in Fig. 2. The control signal is calculated by minimizing the difference between the estimated system output (position) and the given desired reference signal over a given horizon while limiting the magnitude of the control signal. Therefore, a model of the robot is required to estimate future outputs based on current inputs and the known reference signal. The reference signal is continually updated, and if the reference signal includes the estimated heart motion as will be shown later, it will be continually updated to reflect any changes in the actual heart motion.

In order to design a GPC law, we begin with a CARIMA model to describe the robot dynamics:

$$
A\left(z^{-1}\right) y[k T]=B\left(z^{-1}\right) u[(k-1) T] z^{-d}+\frac{C\left(z^{-1}\right)}{\Delta} e[k T]
$$

In the above, $z^{-1}$ is the backward shift operator, $\Delta=$ $1-z^{-1}, \mathrm{~T}$ is the sampling interval, and $k$ is an integer. The current system output is $y[k T]$, the previous sampling interval's control signal (input) is $u[(k-1) T]$, and the current disturbance is $e[k T] . A, B$, and $C$ are polynomials of order $n_{a}, n_{b}$, and $n_{c}$ respectively.

$$
\begin{aligned}
& A\left(z^{-1}\right)=1+a_{1} z^{-1}+a_{2} z^{-1}+\ldots+a_{n_{a}} z^{-1} \\
& B\left(z^{-1}\right)=1+b_{1} z^{-1}+b_{2} z^{-1}+\ldots+b_{n_{b}} z^{-1} \\
& C\left(z^{-1}\right)=1+c_{1} z^{-1}+c_{2} z^{-1}+\ldots+c_{n_{c}} z^{-1}
\end{aligned}
$$

The goal of generalized predictive control is to minimize the difference between the optimal prediction of the plant's future outputs given the current output $\hat{y}[(k+j) T \mid k T]$ and the future reference values $w[(k+j) T]$ while keeping the change in the control effort $\Delta u[(k+j-1) T]$ small. The cost function is then:

$$
\begin{array}{r}
J\left(N_{1}, N_{2}, N_{u}\right)=\sum_{j=1}^{N_{u}} \lambda(j)[\Delta u[(k+j-1) T]]^{2}+ \\
\sum_{j=N_{1}}^{N_{2}} \delta(j)[\hat{y}[(k+j) T \mid k T]-w[(k+j) T]]^{2}
\end{array}
$$

where $N_{1}$ and $N_{2}$ are the minimum and maximum costing horizons, $N_{u}$ is the control horizon and is equal to $N_{1}-N_{2}$, and $\delta(j)$ and $\lambda(j)$ are weighting factors. To estimate future values of $y$ we start with the following Diophatine equation:

$$
1=E_{j}\left(z^{-1}\right) \tilde{A}\left(z^{-1}\right)+z^{-j} F_{j}\left(z^{-1}\right)
$$

where $\tilde{A}\left(z^{-1}\right)=\Delta A\left(z^{-1}\right)$, and $E_{j}\left(z^{-1}\right)$ and $F_{j}\left(z^{-1}\right)$ are polynomials of degree $j-1$ and $n_{a}$, respectively. $E_{j}\left(z^{-1}\right)$ and $F_{j}\left(z^{-1}\right)$ can be uniquely found given $A\left(z^{-1}\right)$. Now multiply (1) by $\Delta E_{j}\left(z^{-1}\right) z^{j}$, considering (4), and the white noise case where $C\left(z^{-1}\right)=1$ we obtain:

$$
\begin{aligned}
& \hat{y}[(k+j) T]=F_{j}\left(z^{-1}\right) y[k T]+E_{j}\left(z^{-1}\right) e[(k+j) T] \\
& \quad+E_{j}\left(z^{-1}\right) B\left(z^{-1}\right) \Delta u[(k+j-d-1) T]
\end{aligned}
$$

Assuming the best estimate of future errors $e[(k+j) T]$ is zero we obtain:

$$
\begin{aligned}
& \hat{y}[(k+j) T \mid k T]= \\
& \quad F_{j}\left(z^{-1}\right) y[k T]+G_{j}\left(z^{-1}\right) \Delta u[(k+j-d-1) T]
\end{aligned}
$$

where $G_{j}\left(z^{-1}\right)=E_{j}\left(z^{-1}\right) B\left(z^{-1}\right)$.

In vector form (6) is:

$\mathbf{y}=\mathbf{G u}+\mathbf{F}\left(z^{-1}\right) y[k T]+\mathbf{G}^{\prime}\left(z^{-1}\right) \Delta u[(k-1) T]=\mathbf{G u}+\mathbf{f}$

where

$$
\mathbf{y}=\left[\begin{array}{c}
\hat{y}[(k+d+1) T \mid k T] \\
\hat{y}[(k+d+2) T \mid k T] \\
\vdots \\
\hat{y}\left[\left(k+d+N_{u}\right) T \mid k T\right]
\end{array}\right]
$$

$$
\mathbf{G}=\left[\begin{array}{cccc}
g_{0} & 0 & \ldots & 0 \\
g_{1} & g_{0} & \ldots & 0 \\
\vdots & \vdots & \vdots & \vdots \\
g_{N_{u}-1} & g_{N_{u}-2} & \cdots & g_{0}
\end{array}\right]
$$

$g_{0}, g_{1}, \ldots, g_{N_{u}-1}$ are the coefficients of $G_{j}$,

$$
\mathbf{u}=\left[\begin{array}{c}
\Delta u[k T] \\
\Delta u[(k+1) T] \\
\vdots \\
\Delta u\left[\left(k+N_{u}-1\right) T\right]
\end{array}\right]
$$

$$
\mathbf{G}^{\prime}\left(z^{-1}\right)=
$$

$$
\left[\begin{array}{c}
\left(G_{d+1}\left(z^{-1}\right)-g_{0}\right) z \\
\left(G_{d+2}\left(z^{-1}\right)-g_{0}-g_{1} z^{-1}\right) z^{2} \\
\vdots \\
\left(G_{d+N_{u}}\left(z^{-1}\right)-g_{0}-g_{1} z^{-1}-\ldots g_{N_{u}-1} z^{N_{u}-1}\right) z^{N}
\end{array}\right]
$$




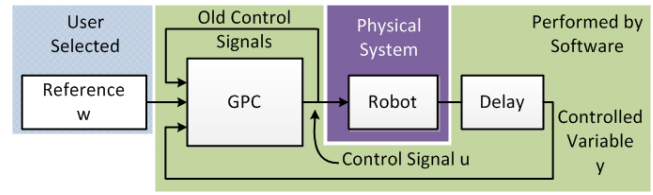

Fig. 2. A GPC feedback control system.

and

$$
\mathbf{F}\left(z^{-1}\right)=\left[\begin{array}{c}
F_{d+1}\left(z^{-1}\right) \\
F_{d+2}\left(z^{-1}\right) \\
\vdots \\
F_{d+N_{u}}\left(z^{-1}\right)
\end{array}\right]
$$

The cost function can be rewritten as:

$$
J=(\mathbf{G u}+\mathbf{f}-\mathbf{w})^{T}(\mathbf{G u}+\mathbf{f}-\mathbf{w})+\lambda \mathbf{u}^{T} \mathbf{u}
$$

where

$$
\mathbf{w}=\left[\begin{array}{c}
w[(k+1) T] \\
\vdots \\
w\left[\left(k+N_{u}\right) T\right]
\end{array}\right]
$$

From (13) $\Delta u[k T]$ can be calculated.

$$
\Delta u[k T]=K(\mathbf{w}-\mathbf{f})
$$

where $\mathbf{K}=\left(\mathbf{G}^{T} \mathbf{G}+\lambda \mathbf{I}\right)^{-1} \mathbf{G}^{T}, I$ is an identity matrix with the same size as $\mathbf{G}, \lambda$ is a weighting factor, and $K$ is the first line of K. For more details see [19].

\section{B. SURGICAL ROBOT CONTROL ALGORITHM}

Two different approaches can be taken when developing the GPC system for a robot-assisted beating-heart surgical system. This choice stems from the fact that there are three possible outputs from the processed images: the heart position, the surgical tool position, and the distance between the heart and the surgical tool. Each of these measurements will be delayed the length of the image acquisition and processing delay. The goal is to make the surgical tool follow the heart's motion and this can be done in two different ways. The first method, shown in Fig. 3, is to control the distance between the surgical tool tip and the heart to follow the surgeon's commanded motion. In this case the reference signal $w$ is simply the surgeon's motion, the controlled variable $y$ is the distance between the surgical tool tip and the heart, and the heart's motion is treated as an unknown disturbance. The second method, shown in Fig. 4, is to directly control the position of the surgical tool to follow the motion of the heart, which is not known in real time. In this research an estimate of the heart position is obtained by delaying the actual heart position by the length of one heart beat. In this case the tracking error is reduced to the difference between the heart motion and the estimated heart motion. The reference signal $w$ is the sum of the surgeon's motion and the estimated heart motion and the controlled variable $y$ is the surgical tool's position. In both figures the darker section is the physical system that cannot be changed and the lighter section is performed by software and can be changed.

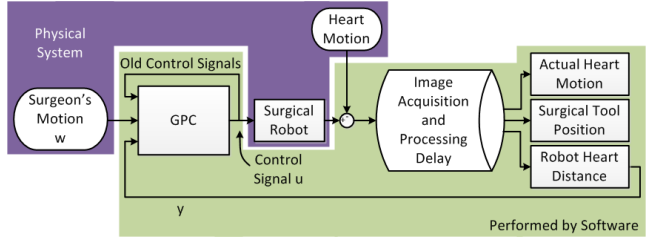

Fig. 3. A model of the robot-assisted beating-heart surgical system where the distance between the surgical tool tip and the POI on the heart is being controlled to follow the surgeon's motion.

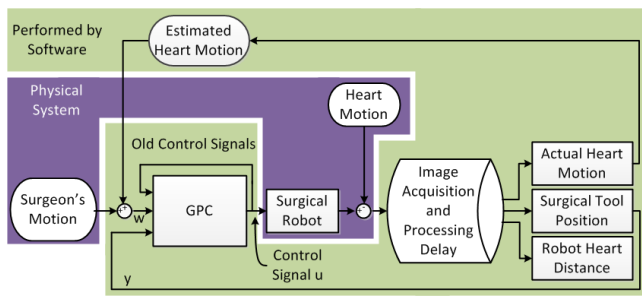

Fig. 4. A model of the robot-assisted beating-heart surgical system where the surgical tool tip is being controlled to follow the combination of the estimated heart motion and the surgeon's motion. The estimated hear motion is calculated from the heart's motion in the previous heart beat.

\section{EXPERIMENTAL RESULTS}

The two proposed GPC control methods were tested experimentally. A voice coil (NCC20-18-020-1X from H2W Technologies Inc., Santa Clarita, CA, USA) shown in Fig.5 was used to actuate the surgical tool. The motion of the surgical tool and a simulated beating heart was captured using an Hx60 MicronTracker from Claron Technology Inc., Toronto, ON, Canada. The MicronTracker was chosen to represent an ultrasound scanner because it has a similar frame rate of $20 \mathrm{~Hz}$, which introduces an image acquisition and processing delay of up to $50 \mathrm{~ms}$. A Yaskawa Motoman Robot SIA5F from Yaskawa Canada, Inc., Mississagua, ON, Canada was used as a beating heart simulator. The experimental setup is shown in Fig. 6 and a close up view of the surgical tool is shown in Fig. 5.

To begin designing a GPC system to control the surgical tool, an electromechanical model of the surgical robot must be identified. This electromechanical model includes the voice coil actuator and the corresponding analog circuitry that converts the voltage setpoint to a current that drives the voice coil actuator. The voice coil actuator was modeled as that in [20] by Oboe et al. where relationship between the input current and the velocity of the voice coil actuator is

$$
V=\left(\frac{1}{s L+R}\right)\left(\frac{K_{t}}{s J+B}\right) \omega
$$

The inductance $L$ and resistance $R$ of the voice coil are $1.05 \mathrm{mH}$ and $3.5 \Omega$, respectively, and the back EMF gain $K_{t}$ is $6.1 \mathrm{~V} / \mathrm{m}$. The parameters $J$ and $B$ were found to be -0.5264 and 16.2547 using least squares identification.

Next, consistent with past literature [17] the Motoman robot was programmed to move linearly in one direction in the same fashion as the heart - see Heart Position in Figs. $7-$ 11. The Micron Tracker captured the motion of the Claron 


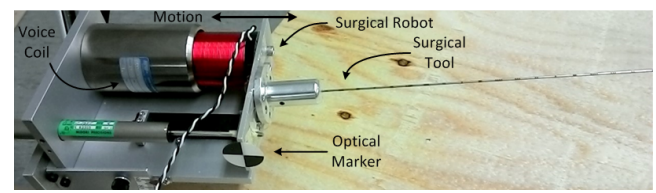

Fig. 5. A close up view of the surgical tool. It is actuated by a voice coil. An optical marker is attached in order for the Micron tracker to measure its position.

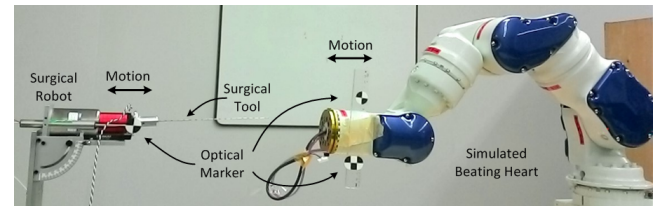

Fig. 6. The experimental setup. The surgical tool is actuated by a voice coil actuator and the beating heart is simulated by an oscillating robotic arm.

black and white optical markers that were attached to the robot arm and to the surgical tool. These markers will not be needed later when the system uses an ultrasound scanner to measure the motion. Each of the two methods of designing the GPC described in Section III-B are implemented. The control horizon was set to 3 and $\lambda=0.00001$.

First, the distance between the surgical tool and the heart is controlled to follow the surgeon's position. There is a $50 \mathrm{~ms}$ delay in the loop due to image acquisition and processing and the images are acquired at a rate of $20 \mathrm{~Hz}$. The heart's motion is treated as a disturbance whose measurement is available after the time delay. The result when the surgeon's motion is set to zero is shown in Fig. 7 where the dashed blue line is the position of the simulated heart and the solid black line is the position of the surgical tool tip. The mean value of the position error is $12 \mathrm{~mm}$ and the integrated squared error (ISE)

$$
I S E=\frac{1}{n} \sum \epsilon^{2}
$$

where $\epsilon$ is the position error and $n$ is the number of data points, is $205 \mathrm{~mm}^{2}$. The result when the surgeon's motion is a square wave with an amplitude of $3 \mathrm{~mm}$ and a frequency of $2 \mathrm{~Hz}$ is shown in Fig. 8 where the dashed blue line is the position of the simulated heart, the solid black line is the position of the surgical tool tip, and the dash-dotted black line is the surgeon's motion. The mean value of the position error is $8.9 \mathrm{~mm}$ and the ISE is $115 \mathrm{~mm}^{2}$. The errors are large because the system is not given any information about the disturbance (simulated heart motion) which has an amplitude of $20 \mathrm{~mm}$. Because of the time delay, the surgical tool tip moves to correct for the heart's motion with a delay.

Second, the surgical tool tip is controlled to follow the combination of the estimated heart motion and the surgeon's motion. As a base for comparison, the surgical tool was first controlled to follow the simulated heart motion under no delay using a proportional controller. The result is given in Fig. 9. It is evident from this figure that the voice coil, under the low sampling rate of $20 \mathrm{~Hz}$, follows the motion with a

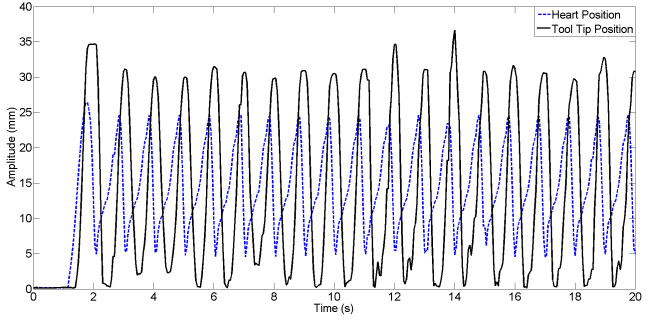

Fig. 7. The result of controlling the surgical tool to keep a fixed distance between itself and the beating heart. In this case the surgeon's motion is set to zero. The diagram of this system is given in Fig. 3 .

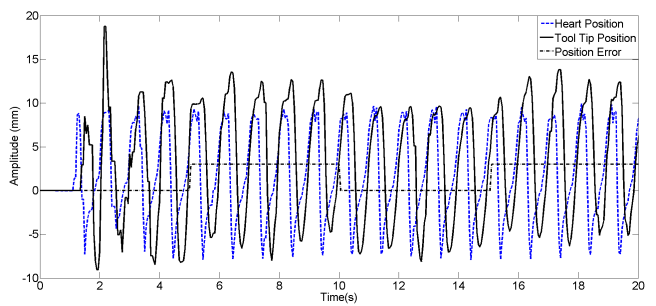

Fig. 8. The result of controlling the surgical tool to keep a fixed distance between itself and the beating heart. In this case the surgeon's motion is a square wave with an amplitude of $3 \mathrm{~mm}$ and a frequency of $2 \mathrm{~Hz}$. The diagram of this system is given in Fig. 3 .

delay. Next the delay was returned and the GPC controller was returned to the system. As before, there is a $50 \mathrm{~ms}$ delay in the system due to image acquisition and processing before the position of the heart is known. For simplicity, the heart's motion is delayed by one heartbeat cycle and then used as a estimate of the current heart position. Now the tracking error is the difference between the current and estimated heart motion and the GPC does not have to reject a large disturbance as in the previous case. The result when the surgeon's motion is set to zero is shown in Fig. 10 where the dashed blue line is the position of the simulated heart and the solid black line is the position of the surgical tool tip. The mean value of the position error is $8.1 \mathrm{~mm}$ and the ISE is $91 \mathrm{~mm}^{2}$. The result when the surgeon's motion is a square wave with an amplitude of $3 \mathrm{~mm}$ and a frequency of $2 \mathrm{~Hz}$ is given in Fig. 11 where the dashed blue line is the position of the simulated heart, the solid black line is the position of the surgical tool tip, and the dash-dotted black line is the surgeon's motion. The mean value of the position error is $8.1 \mathrm{~mm}$ and the ISE is $91 \mathrm{~mm}^{2}$. No additional error occurred when the surgeon's motion was added. These position errors are smaller than those in the previous method, showing the superiority of the second method.

\section{CONCLUDING REMARKS}

Developing a surgical system that controls a surgical tool to follow the combination of the heart's and the surgeon's motion is a challenging task for many reasons. The first being that the heart's motion and the surgical tool's motion are only available after the image acquisition and processing delay. If ultrasound images are used (in the case of this paper camera images with delays similar to ultrasound images), 


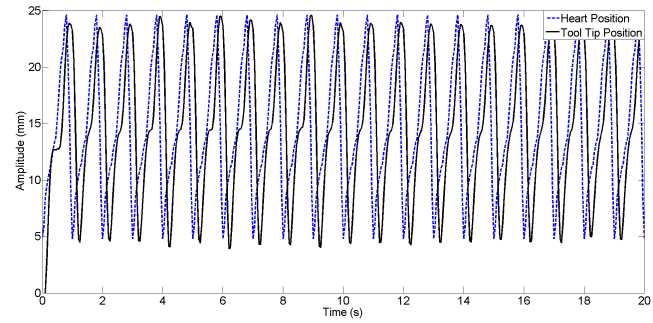

Fig. 9. The result of controlling the surgical tool to follow the simulated heart motion under no delay using proportional control.

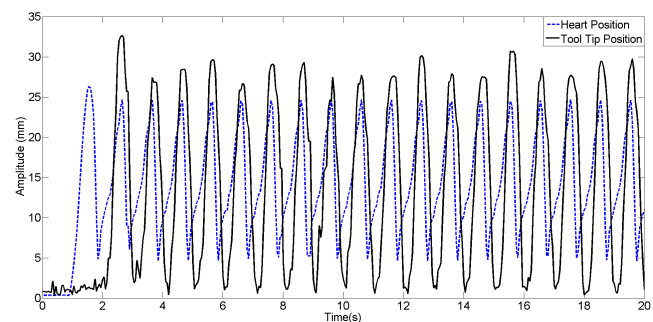

Fig. 10. The result of controlling the surgical tool to follow the estimated heart position. In this case the surgeon's motion is set to zero. The diagram of this system is given in Fig. 4 .

these delays can be larger than $50 \mathrm{~ms}$. However, ultrasound can visualize through blood and is therefore necessary for intracardiac procedures. Secondly, the surgical tool must follow the combination of the surgeon's motion, which is available in real-time, and the heart's motion, which is not available in real-time. The heart's motion can be treated as a disturbance that the control system must reject, but this is difficult as the heart moves quickly with velocities up to $210 \mathrm{~mm} / \mathrm{s}$ and accelerations up to $3800 \mathrm{~mm} / \mathrm{s}^{2}$. In this paper a generalized predictive controller was implemented in two different manners to make the surgical tool follow the combination of the heart's and surgeon's motion. The first treated the heart's motion as a disturbance and controlled the distance between the heart and surgical tool to follow the surgeon's motion. The second method estimated the heart's motion based on previous motion and controlled the surgical tool to follow the combination of the surgeon's motion and the estimated heart motion. Because a large disturbance was not present in the second GPC method, the mean error was reduced to $8.1 \mathrm{~mm}$ from $12 \mathrm{~mm}$ in the first GPC method.

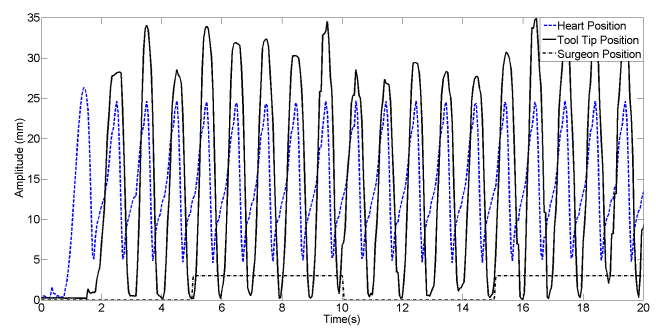

Fig. 11. The result of controlling the surgical tool to follow the estimated heart position. In this case the surgeon's motion is a square wave with an amplitude of $3 \mathrm{~mm}$ and a frequency of $2 \mathrm{~Hz}$. The diagram of this system is given in Fig. 4.
In closing, this paper shows that GPC can be used to create a robot-assisted beating-heart surgical system because it can account for both the image acquisition and processing delay and the large disturbance created by the beating heart.

\section{REFERENCES}

[1] A. Alwan, "Global status report on noncommunicable diseases 2010," World Health Organization, 2011

[2] D. T. Kettler, R. D. Plowes, P. M. Novotny, N. V. Vasilyev, P. J. del Nido, and R. D. Howe, "An active motion compensation instrument for beating heart mitral valve surgery," in IEEE/RSJ Int. Conf. on Intelligent Robots and Systems, 2007, pp. 1290-1295.

[3] T. Ortmaier, M. Groger, D. H. Boehm, V. Falk, and G. Hirzinger, "Motion estimation in beating heart surgery," IEEE Trans. on Biomedical Engineering, vol. 52, no. 10, pp. 1729-1740, 2005.

[4] M. F. Newman, J. L. Kirchner, B. Phillips-Bute, V. Gaver, H. Grocott, R. H. Jones, D. B. Mark, J. G. Reves, and J. A. Blumenthal, "Longitudinal assessment of neurocognitive function after coronaryartery bypass surgery," New England Journal of Medicine, vol. 344, no. 6, pp. 395-402, 02/08 2001.

[5] G. Reed, D. Singer, E. Picard, and R. DeSanctis, "Stroke following coronary-artery bypass surgery. a case-control estimate of the risk from carotid bruits," The New England Journal of Medicine, vol. 319, pp. $1246-1250,1988$.

[6] S. Kesner and R. Howe, "Motion compensated catheter ablation of the beating heart using image guidance and force control," in Int. Symposium on Experimental Robotics, 2012.

[7] M. Joinie-Maurin, B. Bayle, and J. Gangloff, "Force feedback teleoperation with periodical disturbance compensation," in IEEE Int. Conf. on Robotics and Automation, may 2011, pp. 4828 -4833.

[8] B. Cagneau, N. Zemiti, D. Bellot, and G. Morel, "Physiological motion compensation in robotized surgery using force feedback control," in IEEE Int. Conf. on Robotics and Automation, 2007, pp. 1881-1886.

[9] J. Florez, J. Szewczyk, and G. Morel, "An impedance control strategy for a hand-held instrument to compensate for physiological motion," in IEEE Int. Conf. on Robotics and Automation (ICRA), 2012, pp. 1952-1957.

[10] O. Bebek and M. C. Cavusoglu, "Intelligent control algorithms for robotic-assisted beating heart surgery," IEEE Trans. on Robotics, vol. 23, no. 3, pp. 468-480, 2007.

[11] T. Horiuchi, E. Tuna, K. Masamune, and M. Cavusoglu, "Heart motion measurement with three dimensional sonomicrometry and acceleration sensing," in IEEE/RSJ Int. Conf. on Intelligent Robots and Systems, 2012, pp. 4143-4149.

[12] E. Tuna, T. Franke, O. Bebek, A. Shiose, K. Fukamachi, and M. Cavusoglu, "Heart motion prediction based on adaptive estimation algorithms for robotic-assisted beating heart surgery," IEEE Trans. on Robotics, vol. 29, no. 1, pp. 261-276, 2013.

[13] R. Ginhoux, J. Gangloff, M. de Mathelin, L. Soler, M. M. A. Sanchez, and J. Marescaux, "Active filtering of physiological motion in robotized surgery using predictive control," IEEE Trans. on Robotics, vol. 21 , no. 1, p. 67,2005 .

[14] Y. Nakamura, K. Kishi, and H. Kawakami, "Heartbeat synchronization for robotic cardiac surgery," in IEEE Int. Conf. on Robotics and Automation, vol. 2, 2001, pp. 2014-2019 vol.2.

[15] M. Bowthorpe, M. Tavakoli, , H. Becher, and R. Howe, "Smith predictor based robot control ultrasound-guided teleoperated beatingheart surgery," IEEE Journal of Biomedical and Health Informatics, 2013, In press.

[16] P. M. Novotny, J. A. Stoll, P. E. Dupont, and R. D. Howe, "Realtime visual servoing of a robot using three-dimensional ultrasound," in IEEE Int. Conf. on Robotics and Automation, 2007, pp. 2655-2660.

[17] S. G. Yuen, P. M. Novotny, and R. D. Howe, "Quasiperiodic predictive filtering for robot-assisted beating heart surgery," in IEEE Int. Conf. on Robotics and Automation, 2008, pp. 3875-3880.

[18] D. Clarke, C. Mohtadi, and P. Tuffs, "Generalized predictive control part i. the basic algorithm," Automatica, vol. 23, no. 2, pp. 137 - 148, 1987.

[19] E. F. Camacho and C. Bordons, Model Predictive Control, M. J. Grimble and M. A. Johnson, Eds. Springer, 1999.

[20] R. Oboe, F. Marcassa, and G. Maiocchi, "Hard disk drive with voltagedriven voice coil motor and model-based control," IEEE Trans. on Magnetics, vol. 41, no. 2, pp. 784-790, 2005. 